\title{
An Implementation of the PCP, SRP, D-PCP, M-PCP, and FMLP Real-Time Synchronization Protocols in LITMUS ${ }^{\mathrm{RT}}$
}

\author{
Björn B. Brandenburg and James H. Anderson \\ The University of North Carolina at Chapel Hill
}

\begin{abstract}
We extend the FMLP to partitioned static-priority scheduling and derive corresponding worst-case blocking bounds. Further, we present the first implementation of the $P C P$, $S R P, D-P C P, M-P C P$, and FMLP synchronization protocols in a unified framework in a general-purpose OS and discuss design issues that were beyond the scope of prior algorithmic-oriented work on real-time synchronization.
\end{abstract}

\section{Introduction}

With the continued push towards multicore architectures by most (if not all) major chip manufacturers [22, 28], the computing industry is facing a paradigm shift: in the near future, multiprocessors will be the norm. While current offthe-shelf systems already routinely contain processors with two, four, and even eight cores (examples include the Intel Core 2 Duo, the AMD Phenom, and SUN UltraSPARC T1 processors), systems with up to 80 cores are projected to become available within a decade [28]. Not surprisingly, with multicore platforms so widespread, (soft) real-time applications are already being deployed on them. For example, systems processing time-sensitive business transactions have been realized by Azul Systems on top of the highly-parallel Vega2 platform, which consists of up to 768 cores [5].

Motivated by these developments, research on multiprocessor real-time systems has intensified in recent years (see [15] for a survey), with significant effort being focused on both soft and hard real-time scheduling and synchronization [16, 21]. So far, however, few proposed approaches have actually been implemented in operating systems and evaluated under real-world conditions.

In an effort to help bridge the gap between algorithmic research and real-world systems, our group recently developed LITMUS $^{\mathrm{RT}}$, a multiprocessor real-time extension of Linux $[9,13]$. The development of LITMUS ${ }^{\mathrm{RT}}$ has occurred at an auspicious time, given the increasing interest in real-time variants of Linux (see, for example, [1]). These variants will undoubtedly be ported to multicore platforms and thus could benefit from recent algorithmic advances in scheduling-related research. LITMUS ${ }^{\mathrm{RT}}$ has been used to assess the performance of various dynamicpriority scheduling policies with real-world overheads considered [13]. More recently, a study was conducted to com- pare synchronization alternatives under global and partitioned earliest-deadline-first (EDF) scheduling [10].

The versions of LITMUS ${ }^{\mathrm{RT}}$ published so far have exclusively focused on dynamic-priority scheduling algorithms. In this paper, we extend this work by presenting an integrated implementation that supports five major real-time synchronization algorithms under partitioned static-priority (P-SP) scheduling. To our knowledge, this is the first such implementation effort to be conducted on a modern generalpurpose multiprocessor operating system. Moreover, including support for P-SP scheduling in LITMUS ${ }^{\mathrm{RT}}$ is important, as static-priority scheduling is widely used.

Prior Work. Sha et al. were the first to propose protocols for uniprocessors to bound priority inversion - the priority inheritance protocol - and also avoid deadlock - the priority ceiling protocol (PCP) [27]. As an alternative to the PCP, Baker proposed the stack resource policy (SRP) [4]. Both the SRP and the PCP have received considerable attention and have been applied to both EDF and rate monotonic (RM) scheduling.

Rajkumar et al. presented two extensions of the PCP for multiprocessor real-time systems under partitioned staticpriority scheduling: the distributed priority ceiling protocol (D-PCP) [26], which does not require shared memory and thus can be used in distributed systems as well as tightlycoupled multiprocessors, and the multiprocessor priority ceiling protocol (M-PCP) [24], which relies on globallyshared semaphores.

Several multiprocessor synchronization protocols have been proposed for partitioned EDF scheduling. Chen and Tripathi [14] proposed a solution that only applies to synchronous periodic tasks. Additionally, multiprocessor extensions of the SRP for partitioned EDF were proposed by Lopez et al. [21] and Gai et al. [16]. Given the experimental focus of this paper, it is worth noting that Gai et al. not only introduced a new locking protocol, the multiprocessor stack resource policy (M-SRP), but also discussed an implementation of it. Their study showed that the M-SRP outperforms the M-PCP. In recent work, Block et al. proposed the flexible multiprocessor locking protocol (FMLP) for both global and partitioned EDF and showed that it outperforms the M-SRP [6].

Contributions. The contributions of our work are threefold: (i) we extend the FMLP to P-SP scheduling and derive corresponding worst-case blocking bounds; (ii) we 
present and discuss in detail the first implementation of the SRP, PCP, M-PCP, D-PCP, and FMLP in one unified framework (which is available publicly under an open source license [17] and, we hope, will serve as a guide for practitioners); and (iii) we discuss implementation and software design issues not fully considered in earlier algorithmic-oriented work on real-time locking protocols.

The rest of this paper is organized as follows: Sec. 2 provides an overview of needed background, Sec. 3 presents the FMLP for P-SP, Sec. 4 discusses the implementation of the synchronization protocols listed above in LITMUS ${ }^{\mathrm{RT}}$, and Sec. 5 concludes. Bounds for worst-case blocking under the FMLP are derived in Appendix A.

\section{Background}

In this section, we describe background necessary for discussing the implementation of the aforementioned synchronization protocols in LITMUS ${ }^{\mathrm{RT}}$.

\subsection{System Model}

In this paper, we consider the problem of scheduling a system $T$ of sporadic tasks that share resources upon a multiprocessor platform consisting of $m$ identical processors. A sporadic task $T_{i}$ releases a sequence of jobs $T_{i}^{j}$ and is characterized by its worst-case execution cost, $\mathrm{e}\left(T_{i}\right)$, and its period, $\mathrm{p}\left(T_{i}\right)$. A job $T_{i}^{j}$ becomes available for execution at its release time, $\mathrm{r}\left(T_{i}^{j}\right)$, and should complete execution before its absolute deadline, $\mathrm{d}\left(T_{i}^{j}\right)=\mathrm{r}\left(T_{i}^{j}\right)+\mathrm{p}\left(T_{i}\right)$. A task $T_{i}$ 's jobs are ordered by release time and must be separated by at least $\mathrm{p}\left(T_{i}\right)$ time units, i.e., $j<k \Leftrightarrow \mathrm{r}\left(T_{i}^{j}\right)+\mathrm{p}\left(T_{i}\right) \leq \mathrm{r}\left(T_{i}^{k}\right)$.

On uniprocessors, both the EDF and the RM policies are commonly used to schedule sporadic task systems [19]. Under EDF, jobs with earlier deadlines have higher priority; under RM, tasks with smaller periods have higher priority.

There are two fundamental approaches to scheduling sporadic tasks on multiprocessors - global and partitioned. With global scheduling, processors are scheduled by selecting jobs from a single, shared queue, whereas with partitioned scheduling, each processor has a private queue and is scheduled independently using a uniprocessor scheduling policy (hybrid approaches exist, too [12]). Tasks are statically assigned to processors under partitioning. As a consequence, under partitioned scheduling, all jobs of a task execute on the same processor, whereas migrations may occur in globally-scheduled systems. A discussion of the tradeoffs between global and partitioned scheduling is beyond the scope of this paper and the interested reader is referred to prior studies $[9,13,15]$.

In this paper, we consider only partitioned static-priority (P-SP) scheduling (the use of the FMLP under global and partitioned EDF has been investigated previously $[6,9])$.

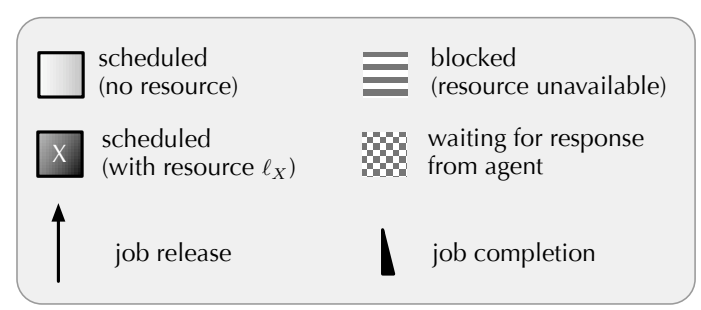

Figure 1: Legend.

Under P-SP, each task is statically assigned to a processor and each processor is scheduled independently using a static-priority uniprocessor algorithm such as RM.

We assume that tasks are indexed from 1 to $n$ by decreasing priority, i.e., a lower index implies higher priority. We refer to $T_{i}$ 's index $i$ as its base priority. A job is scheduled using its effective priority, which can sometimes exceed its base priority under certain resource-sharing policies (e.g., priority inheritance may raise a job's effective priority).

After its release, a job $T_{i}^{j}$ is said to be pending until it completes. While it is pending, $T_{i}^{j}$ is either runnable or suspended. A suspended job cannot be scheduled. When a job transitions from suspended to runnable (runnable to suspended), it is said to resume (suspend). While runnable, a job is either preemptable or non-preemptable. A newlyreleased or resuming job $T_{k}^{l}$ can only preempt a scheduled lower-priority job $T_{i}^{j}$ if $T_{i}^{j}$ is preemptable.

Resources. When a job $T_{i}^{j}$ requires a shared resource $\ell$, it issues a request $\mathcal{R}$ for $\ell$. $\mathcal{R}$ is satisfied as soon as $T_{i}^{j}$ holds $\ell$, and completes when $T_{i}^{j}$ releases $\ell .|\mathcal{R}|$ denotes the maximum duration that $T_{i}^{j}$ will hold $\ell$. A resource can only be held by one job at any time. Thus, $T_{i}^{j}$ may become blocked on $\ell$ if $\mathcal{R}$ cannot be satisfied immediately. A resource $\ell$ is local to a processor $p$ if all jobs requesting $\ell$ execute on $p$, and global otherwise.

If $T_{i}^{j}$ issues another request $\mathcal{R}^{\prime}$ before $\mathcal{R}$ is complete, then $\mathcal{R}^{\prime}$ is nested within $\mathcal{R}$. In such cases, $|\mathcal{R}|$ includes the cost of blocking due to requests nested in $\mathcal{R}$. Note that not all synchronization protocols allow nested requests. If allowed, nesting is proper, i.e., $\mathcal{R}^{\prime}$ must complete no later than $\mathcal{R}$ completes. An outermost request is not nested within any other request. Fig. 2 illustrates the different phases of a resource request. In this and later figures, the legend shown in Fig. 1 is assumed.

Resource sharing introduces a number of problems that can endanger temporal correctness. Priority inversion occurs when a high-priority job $T_{h}^{i}$ cannot proceed due to a lower-priority job $T_{l}^{j}$ either being non-preemptable or holding a resource requested by $T_{h}^{i}$. $T_{h}^{i}$ is said to be blocked by $T_{l}^{j}$. Another source of delay is remote blocking, which occurs when a global resource requested by a job is already 


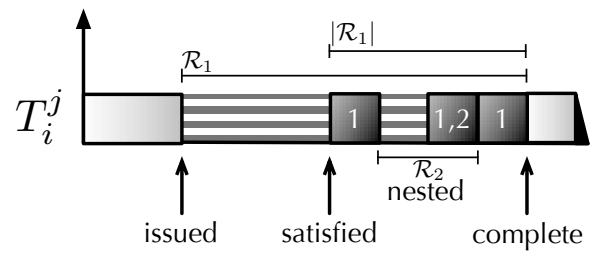

Figure 2: The different phases of a resource request. $T_{i}^{j}$ issues $\mathcal{R}_{1}$ and blocks since $\mathcal{R}_{1}$ is not immediately satisfied. $T_{i}^{j}$ holds $\mathcal{R}_{1} . \ell$ for $\left|\mathcal{R}_{1}\right|$ time units. Note that $\left|\mathcal{R}_{1}\right|$ includes blocking incurred due to nested requests.

in use on another processor. If the maximum duration of priority inversion and remote blocking is not bounded, then timing guarantees cannot be given.

\subsection{Local Synchronization Protocols}

Requests for local resources are arbitrated using uniprocessor synchronization protocols. Such protocols are preferable to global protocols (where applicable) because their worst-case blocking delays are generally shorter. In LITMUS $^{\mathrm{RT}}$, we have implemented both the PCP and the SRP. Note that at most one local protocol can be in use.

The PCP and the SRP both are based on the notion of a priority ceiling. ${ }^{1}$ The priority ceiling of a resource $\ell$ is the highest priority of any job that requests $\ell$. The system ceiling (on processor $p$ ) is the maximum priority ceiling of all (local) resources currently in use. The system ceiling is $\infty$ if none are in use (on processor $p$ ).

Under the PCP, the system ceiling is used to arbitrate (local) resource requests directly. When a job $T_{i}^{j}$ requests a resource, $T_{i}^{j}$ 's priority is compared to the current system ceiling. If $T_{i}^{j}$ 's priority exceeds the system ceiling (or if $T_{i}^{j}$ holds the resource that raised the system ceiling last), then the request is satisfied, otherwise $T_{i}^{j}$ suspends. The PCP also uses priority inheritance - while a lower-priority job $T_{k}^{l}$ blocks a higher-priority job $T_{i}^{j}$ (directly or indirectly), $T_{k}^{l}$ 's effective priority is raised to (at least) $T_{i}^{j}$ 's effective priority. Note that priority inheritance is transitive.

Under the SRP, resource requests are always satisfied immediately. Blocking only occurs on release - a job $T_{i}^{j}$ may not execute after its release until its priority exceeds the system ceiling. Thus, jobs are blocked at most once and there is no need for priority inheritance. (If jobs suspend, then they can also block each time they resume.)

The nesting of local resources is permitted under both the PCP and the SRP. Both protocols avoid deadlock and bound the maximum length of priority inversions [4, 27].

\footnotetext{
${ }^{1}$ This section is intended as a brief reminder and assumes familiarity with the discussed protocols. For a full discussion, the interested reader is referred to [20].
}

Example. In Fig. 3, two schedules for three resourcesharing jobs are shown. Inset (a) depicts resource sharing under the PCP. $T_{3}^{1}$ issues a request for $\mathcal{R}_{1}$ at time 1 , which is satisfied immediately. This raises the system ceiling from $\infty$ to two. At time $2, T_{2}^{1}$ is released and preempts $T_{3}^{1} . T_{2}^{1}$ requests $\mathcal{R}_{2}$ at time 4 , but since its priority does not exceed the system ceiling, it becomes blocked and suspends until time 6 when $\mathcal{R}_{1}$ is released, which momentarily lowers the system ceiling to $\infty$. The system ceiling is raised to one again when $T_{2}^{1}$ 's request is satisfied. $T_{1}^{1}$ arrives at time 7 and preempts $T_{2}^{1}$. $T_{1}^{1}$ requests $\mathcal{R}_{2}$ at time 8 and suspends, since the system ceiling is still one. This gives $T_{2}^{1}$ a chance to request $\mathcal{R}_{1}$ (which is satisfied since $T_{2}^{1}$ raised the system ceiling last), to finish its critical section, and to release both $\mathcal{R}_{1}$ and $\mathcal{R}_{2}$ at time 9 . This allows $T_{1}^{1}$ to proceed. Finally, all jobs complete in order of priority.

Inset (b) depicts a similar schedule for the same task system under the SRP. Note that all blocking has been "moved" to occur immediately after a job has been released. For example, when $T_{2}^{1}$ is released at time 2, the current system ceiling is already two. Thus, $T_{2}^{1}$ is blocked until time 4 , when the system ceiling is lowered to $\infty$.

\subsection{Global Synchronization Protocols}

A global synchronization protocol is required if jobs executing on different processors may request a resource concurrently. In this paper (and in the LITMUS ${ }^{\mathrm{RT}}$ kernel), we focus on three global synchronization protocols: the D-PCP, the M-PCP, and the FMLP. The D-PCP and the M-PCP are reviewed next; the FMLP is discussed in greater detail in Sec. 3.

The D-PCP extends the PCP by providing local agents that act on behalf of requesting jobs. A local agent $A_{i}^{q}$, located on remote processor $q$ where jobs of $T_{i}$ request resources, carries out requests on behalf of $T_{i}$ on processor $q$. Instead of accessing a global remote resource $\ell$ on processor $q$ directly, a job $T_{i}^{j}$ submits a request $\mathcal{R}$ to $A_{i}^{q}$ and suspends. $T_{i}^{j}$ resumes when $A_{i}^{q}$ has completed $\mathcal{R}$. To expedite requests, $A_{i}^{q}$ executes with an effective priority higher than that of any normal task (see [20, 25] for details). However, agents of lower-priority tasks can still be preempted by agents of higher-priority tasks. When accessing global resources residing on $T_{i}$ 's assigned processor, $T_{i}^{j}$ serves as its own agent. Note that, because jobs do not access remote global resources directly, the D-PCP is suitable for use in distributed systems where processors do not share memory.

The M-PCP is an extension of the PCP that relies on shared memory to support global resources. In contrast to the D-PCP, global resources are not assigned to any particular processor but are accessed directly. Local agents are not required since jobs execute requests themselves on their assigned processors. Competing requests are satisfied in or- 


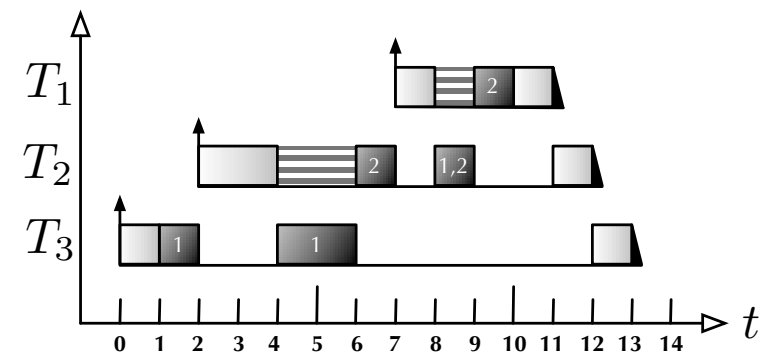

(a)

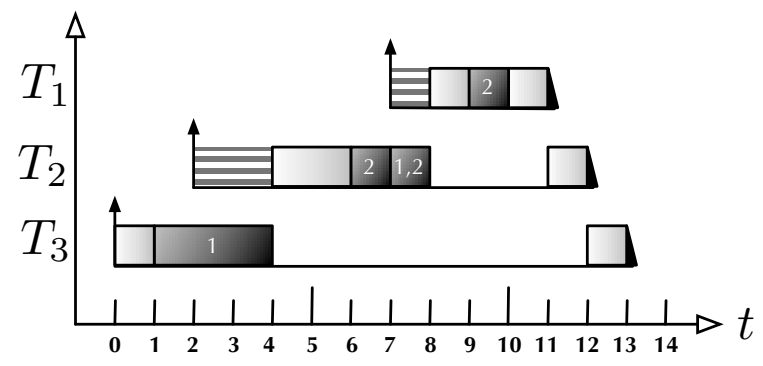

(b)

Figure 3: Two example schedules in which three tasks share two local resources (only initial jobs shown, deadlines omitted). The priority ceiling of $\mathcal{R}_{1}$ is two, and the priority ceiling of $\mathcal{R}_{2}$ is one. (a) PCP schedule. (b) SRP schedule.

der of job priority. When a request is not satisfied immediately, the requesting job suspends until its request is satisfied. Under the M-PCP, jobs holding global resources execute with an effective priority higher than that of any normal task.

Both the D-PCP and the M-PCP avoid global deadlock by prohibiting the nesting of global resource requests a global request $\mathcal{R}$ cannot be nested within another request (either local or global) and no other request (local or global) may be nested within $\mathcal{R}$.

Example. Fig. 4 depicts global schedules for four jobs $\left(T_{1}^{1}, \ldots, T_{4}^{1}\right)$ sharing two resources $\left(\ell_{1}, \ell_{2}\right)$ on two processors. Inset (a) shows resource sharing under the D-PCP. Both resources reside on processor 1 . Thus, two agents $\left(A_{2}^{1}\right.$, $A_{4}^{1}$ ) are also assigned to processor 1 in order to act on behalf of $T_{2}$ and $T_{4}$ on processor 2. $A_{4}^{1}$ becomes active at time 2 when $T_{4}^{1}$ requests $\ell_{1}$. However, since $T_{3}^{1}$ already holds $\ell_{1}$, $A_{4}^{1}$ is blocked. Similarly, $A_{2}^{1}$ becomes active and blocks at time 4 . When $T_{3}^{1}$ releases $\ell_{1}, A_{2}^{1}$ gains access next because it is the highest-priority active agent on processor 1 . Note that, even though the highest-priority job $T_{1}^{1}$ is released at time 2, it is not scheduled until time 7 because agents and resource-holding jobs have an effective priority that exceeds the base priority of $T_{1}^{1} . A_{2}^{1}$ becomes active at time 9 since $T_{2}^{1}$ requests $\ell_{2}$. However, $T_{1}^{1}$ is accessing $\ell_{1}$ at the time, and thus has an effective priority that exceeds $A_{2}^{1}$ 's priority. Therefore, $A_{2}^{1}$ is not scheduled until time 10.

Inset (b) shows the same scenario under the M-PCP. Local agents are no longer required since $T_{2}^{1}$ and $T_{4}^{1}$ access global resources directly. $T_{4}^{1}$ suspends at time 2 since $T_{3}^{1}$ already holds $\ell_{1}$. Similarly, $T_{2}^{1}$ suspends at time 4 until it holds $\ell_{1}$ one time unit later. Meanwhile, on processor 1, $T_{1}^{1}$ is scheduled at time 5 after $T_{2}^{1}$ returns to normal priority and also requests $\ell_{1}$ at time 6 . Since resource requests are satisfied in priority order, $T_{1}^{1}$ 's request has precedence over $T_{4}^{1}$,s request, which was issued much earlier at time 2 . Thus, $T_{4}^{1}$ must wait until time 8 to access $\ell_{1}$. Note that $T_{4}^{1}$ preempts $T_{2}^{1}$ when it resumes at time 8 since it is holding a global resource.

\section{The FMLP under P-SP}

The flexible multiprocessor locking protocol (FMLP) is a global real-time synchronization protocol that was recently proposed by Block et al. [6]. It is intended to overcome shortcomings of prior protocols such as the inability to nest resources and overly pessimistic analysis. Block et al. originally proposed the FMLP for global and partitioned EDF. In this paper, we show how to adapt the FMLP to P-SP scheduling.

\subsection{Design Choices}

The FMLP is based on two fundamental design principles - flexibility and simplicity. We desire flexibility so as to not unnecessarily restrict the range of options available to application designers. We favor simple mechanisms because they allow us to bound worst-case scenarios more tightly. The latter is especially critical - our ability to analyze a real-time system is more important than raw performance. Based on these two principles, the FMLP was originally designed - and adapted for P-SP here - by focusing on three issues that every global synchronization protocol must address: how to block, how to limit remote blocking, and how to handle nested requests.

Blocking. When a resource request cannot be satisfied immediately, the requesting job cannot proceed to execute: it is blocked. On a multiprocessor, there are two ways to handle such a situation. The blocked job can either remain scheduled and busy-waits until its request is satisfied, or it can relinquish its processor and let other jobs execute while it is suspended. Traditionally, busy-waiting has mostly been used in scenarios where resources are held only for very short times, since busy-waiting clearly wastes processing capacity. (Under the D-PCP and the M-PCP, jobs block by suspending.) However, recent studies have shown that, for real-time systems, busy-waiting is often preferable [10]. In the interest of flexibility, the FMLP allows both.

In the FMLP, global resources are classified as either 


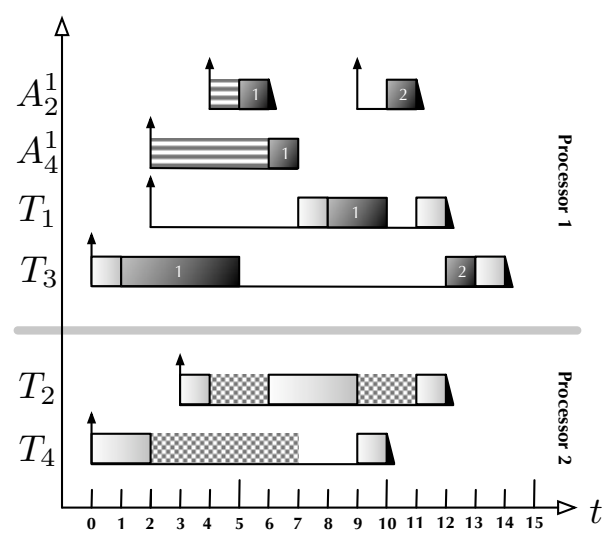

(a)

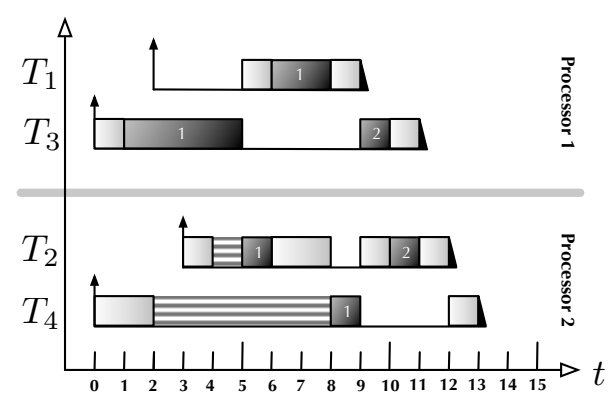

(b)

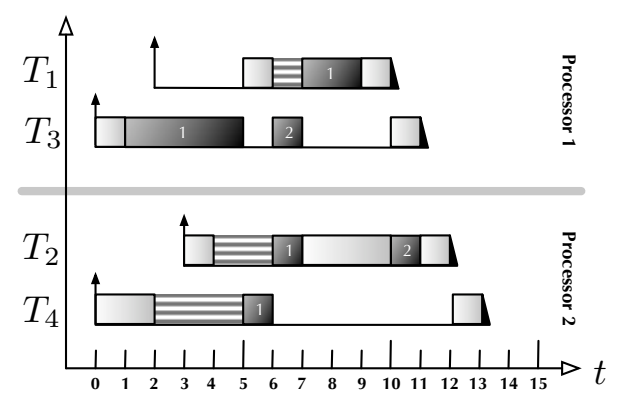

(c)

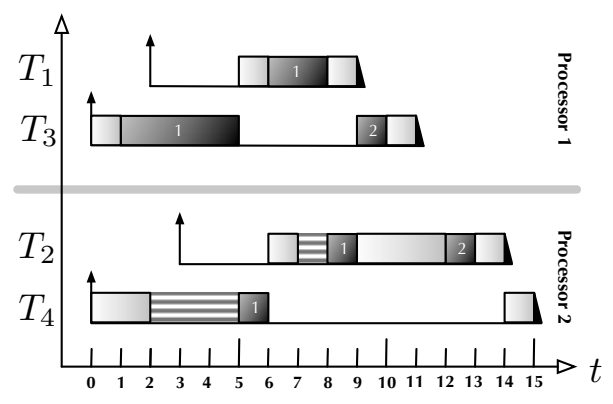

(d)

Figure 4: Example schedules of four tasks sharing two global resources. (a) D-PCP schedule. (b) M-PCP schedule. (c) FMLP schedule $\left(\ell_{1}, \ell_{2}\right.$ are long). (d) FMLP schedule $\left(\ell_{1}, \ell_{2}\right.$ are short).

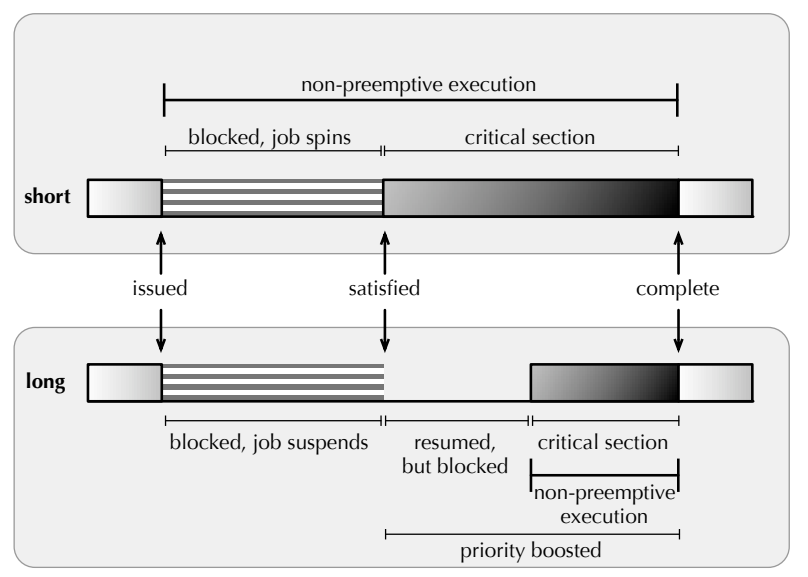

Figure 5: The phases of short and long resource requests.

short or long - tasks busy-wait when blocked on short resources and suspend when blocked on long resources. Resources are classified by the application designer. However, requests for long resources cannot be nested within requests for short resources.

Remote blocking. When all tasks are independent, processors can be analyzed individually (under partitioning). In the presence of globally-shared resources, remote blocking may occur. As a result, processors are no longer independent and potentially pessimistic assumptions must be made to bound worst-case delays. To minimize the impact of remote blocking, resource-holding jobs should complete their requests as quickly as possible. The D-PCP and M-PCP expedite the completion of requests by letting resourceholding jobs (or agents) execute at elevated priorities that exceed normal job priorities - a resource-holding job cannot be preempted by a job that does not hold a resource. However, preemptions may occur among resource-holding jobs (and agents). The FMLP uses a simplified approach. To minimize the delay a job experiences when resuming, the FMLP boosts the priority of resuming jobs equally a resource-holding job is scheduled with effective priority 0 to preempt any non-resource-holding job. Contending priority-boosted jobs are scheduled on a FIFO basis. (Note that priority boosting was not used in prior FMLP variants.) Additionally, to avoid delays due to preemptions, all requests (both short and long) are executed non-preemptively, i.e., a job that executes a request cannot be preempted by any other job. Note that, in the case of short resources, spinning is carried out non-preemptively, too. Priority boosting is not required for short resources since requesting jobs do not suspend when blocked. Fig. 5 illustrates the differences between long and short requests.

Nesting. Nested resource requests may lead to deadlock and negatively affect worst-case delay bounds. To avoid these problems, the D-PCP and the M-PCP disallow nest- 
ing (for global resources) altogether. However, nesting does occur in practice (albeit infrequently) [8]. The FMLP strikes a balance between supporting nesting and optimizing for the common case (no nesting) by organizing resources into resource groups, which are sets of resources (either short or long, but not both) that may be requested together. Two resources are in the same group iff there exists a job that requests both resources at the same time. We let $\mathrm{G}(\ell)$ denote the group that contains $\ell$. Each group is protected by a group lock, which is either a non-preemptive queue lock [3] (for a group of short resources) or a semaphore (for a group of long resources). Under the FMLP, a job always acquires a resource's group lock before accessing the resource. Note that, with the introduction of groups, the term "outermost" is interpreted with respect to groups. Thus, a short resource request that is nested within a long resource request but not within any short resource request is considered to be outermost.

Fig. 6 shows an example wherein seven resources (two long, five short) are grouped into three resource groups. Note that, even though a request for $\ell_{2}^{l}$ may contain a request for $\ell_{7}^{s}$, the two resources belong to different groups since one is short and one is long.

\subsection{Request Rules}

Based on the discussion above, we now define the rules for how resources are requested in the FMLP under P-SP scheduling.

We assume that resources have been grouped appropriately beforehand, and that non-preemptive sections can be nested, i.e., if a job enters a non-preemptive section while being non-preemptive, then it only becomes preemptable after leaving the outermost non-preemptive section. Let $T_{i}^{j}$ be a job that issues a request $\mathcal{R}$ for resource $\ell$. First, we only consider outermost requests.

Short requests. If $\mathcal{R}$ is short and outermost, then $T_{i}^{j}$ becomes non-preemptable and attempts to acquire the queue lock protecting $\mathrm{G}(\ell)$. In a queue lock, blocked processes busy-wait in FIFO order. $\mathcal{R}$ is satisfied once $T_{i}^{j}$ holds $\ell$ 's group lock. When $\mathcal{R}$ completes, $T_{i}^{j}$ releases the group lock and leaves its non-preemptive section.

Long requests. If $\mathcal{R}$ is long and outermost, then $T_{i}^{j}$ attempts to acquire the semaphore protecting $\mathrm{G}(\ell)$. Under a semaphore lock, blocked jobs are added to a FIFO queue and suspend. As soon as $\mathcal{R}$ is satisfied (i.e., $T_{i}^{j}$ holds $\ell$ 's group lock), $T_{i}^{j}$ resumes (if it suspended) and enters a nonpreemptive section (which becomes effective as soon as $T_{i}^{j}$ is scheduled). When $\mathcal{R}$ completes, $T_{i}^{j}$ releases the group lock and becomes preemptive.

Priority boost. If $\mathcal{R}$ is long and outermost, then $T_{i}^{j}$,s priority is boosted when $\mathcal{R}$ is satisfied (i.e., $T_{i}^{j}$ is scheduled

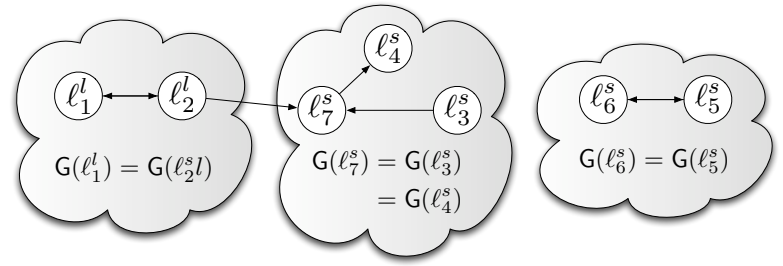

Figure 6: Grouping of two long resources $\left(\ell_{1}^{l}, \ell_{2}^{l}\right)$ and five short resources $\left(\ell_{3}^{s}, \ldots, \ell_{7}^{s}\right)$ under the FMLP. If a request for $\ell$ may contain a request for $\ell^{\prime}$, then this is indicated by a directed edge from $\ell$ to $\ell^{\prime}$.

with effective priority 0 ). This allows it to preempt jobs executing preemptively at base priority. If two or more priorityboosted jobs are ready, then they are scheduled in the order in which their priorities were boosted (FIFO).

Nesting. Nesting is handled in the same manner for long and short resources: when a job $T_{i}^{j}$ issues a request $\mathcal{R}$ for a resource $\ell$ and $T_{i}^{j}$ already holds $\ell$ 's group lock, then $\mathcal{R}$ is satisfied immediately and no further action is taken when $\mathcal{R}$ completes.

Example. Insets (c) and (d) of Fig. 4 depict FMLP schedules for the same scenario previously considered in the context of the D-PCP and the M-PCP. In (c), $\ell_{1}$ and $\ell_{2}$ are classified as long resources. As before, $T_{3}^{1}$ requests $\ell_{1}$ first and forces the jobs on processor 2 to suspend $\left(T_{4}^{1}\right.$ at time 2 and $T_{2}^{1}$ at time 4 ). In contrast to both the D-PCP and the M-PCP, contending requests are satisfied in FIFO order. Thus, when $T_{3}^{1}$ releases $\ell_{1}$ at time $5, T_{4}^{1}$ 's request is satisfied before that of $T_{2}^{1}$. Similarly, $T_{1}^{1}$ 's request for $\ell_{1}$ is only satisfied after $T_{2}^{1}$ completes its request at time 7 . Note that, since jobs suspend when blocked on a long resource, $T_{3}^{1}$ can be scheduled for one time unit at time 6 when $T_{1}^{1}$ blocks on $\ell_{1}$.

Inset (d) depicts the schedule that results when both $\ell_{1}$ and $\ell_{2}$ are short. The main difference to the schedule depicted in (c) is that jobs busy-wait non-preemptively when blocked on a short resource. Thus, when $T_{2}^{1}$ is released at time 3, it cannot be scheduled until time 6 since $T_{4}^{1}$ executed non-preemptively from time 2 until time 6 . Similarly, $T_{4}^{1}$ cannot be scheduled at time 7 when $T_{2}^{1}$ blocks on $\ell_{2}$ because $T_{2}^{1}$ does not suspend. Note that, due to the waste of processing time caused by busy-waiting, the last job only finishes at time 15. Under suspension-based synchronization methods, the last job finishes at either time 13 (M-PCP and FMLP for long resources) or 14 (D-PCP).

Local resources. The FMLP can be integrated with the SRP. When a job blocks at release time due to the SRP, it cannot have requested a global resource yet (and thus does not impact the FMLP analysis). Global short requests can be nested within local requests since jobs do not suspend when blocked on short resources. However, global 
long requests cannot be nested within local requests since a job must not hold local resources when it suspends. Local requests can be nested within global requests since a task never blocks on a local request under the SRP. However, care must be taken to properly account for the interaction between the FMLP and the SRP_- every time a job resumes, it is subject to blocking from local resources.

Properties. The FMLP avoids deadlock - by construction, resources within a group cannot contribute to a deadlock, and the constraint that long requests cannot be nested within short requests prohibits cyclic nesting of resource groups. Bounds for worst-case blocking under the FMLP are derived in Appendix A.

\section{Implementation}

Due to space constraints, we are unable to discuss every detail of each implemented protocol. Instead, we focus on interesting architectural issues that we encountered when designing LITMUS ${ }^{\mathrm{RT}}$. The interested reader is referred to [9], which contains a detailed description of the LITMUS $^{\mathrm{RT}}$ framework and its capabilities and limitations, and to LITMUS ${ }^{\mathrm{RT}}$ 's source code, which is publicly available online [17].

Developed by UNC's real-time group, LITMUS ${ }^{\mathrm{RT}}$ is an extension of Linux that supports a variety of real-time multiprocessor scheduling policies [13]. However, prior to this paper, LITMUS ${ }^{\mathrm{RT}}$ did not support static-priority scheduling. The contribution discussed in this paper is the addition of static-priority scheduling and implementations of the PCP, the D-PCP, the M-PCP, and the FMLP (under P$\mathrm{SP})$ in LITMUS $^{\mathrm{RT}}$.

Real-time Linux. Critics have argued that, due to inherent non-determinism in the kernel's architecture, Linux is fundamentally not capable of providing (hard) real-time guarantees. In practice, however, variants of Linux are increasingly being adopted in (soft) real-time settings [1] the predictability of Linux is sufficient for many applications most of the time. Thus, while no absolute timing guarantees can be given in Linux, it is desirable that neither scheduling nor resource sharing are the weakest links in terms of predictability.

When implemented in a general-purpose OS, real-time algorithms face a real-world requirement that is often glanced over in algorithmic-oriented research - they must degrade gracefully when faced with misbehaving applications. In a real OS, especially during development and testing, jobs may unexpectedly suspend due to page faults, perform diagnostic logging, accidentally request wrong resources, fail to properly deallocate resources, and "get stuck" in non-preemptive sections (among many other possible failures). While real-time guarantees cannot be given for misbehaving jobs, in practice, (partial) resilience to failure is a very desirable property for a well-designed OS. We revisit this issue in more detail in the following paragraphs.

Real-time tasks. A fundamental design decision is how the sporadic task model is mapped onto the Linux process model. In Linux, one or more sequential threads of execution that share an address space are called a process. There are three obvious ways to implement sporadic tasks: (i) a sporadic task is a process, and each job is a thread; (ii) a sporadic task is a thread, and each job is the iteration of a loop; and (iii) a sporadic task is just a concept, and jobs are the invocation of interrupt service routines. Approach (iii), while popular in embedded systems, suffers from a general lack of robustness and the limitations that are imposed on code executing in kernel space (e.g., absence of floating point arithmetic, etc.). Approach (i) suffers from high job release overheads due to forking. This may be alleviated by recycling threads by means of a thread pool, but determining the maximum number of threads required in the face of deadline overruns is non-trivial. Approach (ii) limits how deadline overruns can be handled - late jobs cannot be easily aborted and jobs of the same task cannot be scheduled concurrently. Nonetheless, in LITMUS ${ }^{\mathrm{RT}}$, we chose this approach because it most closely resembles the familiar UNIX programming model. When sporadic tasks are threads, the question arises as to whether all real-time tasks should reside in the same process. From an efficiency point of view, a single-process solution may be beneficial, whereas from a robustness point of view, address space separation is clearly favorable. In LITMUS ${ }^{\mathrm{RT}}$, we support both.

Resource references. Blocking-by-suspending requires kernel support, as does maintaining and enforcing priority ceilings and enacting priority inheritance. Thus, each resource is modeled as an object in kernel space, which contains state information such as the associated priority ceiling, unsatisfied requests, etc. (The exception are short FMLP resources, which are unknown to the kernel, since they are realized almost entirely in user space. See [9] for details.)

All tasks that share a given resource must obtain a reference to the same in-kernel object. Since LITMUS ${ }^{\mathrm{RT}}$ is committed to not unnecessarily restricting the application design space, references must be (transparently) obtainable across process boundaries. For performance reasons, resource references must be resolved by the kernel with as little overhead as possible. Further, in a general-purpose OS such as Linux, security concerns such as visibility of resources and access control must also be addressed - the resource namespace must be managed by the kernel.

Prior versions of LITMUS ${ }^{\mathrm{RT}}$ simply allocated a predefined number of resources statically and let real-time programs refer to objects by their offset. While this interim method had low overheads, it was also completely 
insecure and brittle. Further, the lack of flexibility inherent in static allocation also quickly proved to be troublesome. As part of the FMLP under P-SP implementation effort, we introduced a new solution to manage resources in a secure, reliable, and efficient matter. Instead of introducing a new namespace (which would require appropriate access policies and semantics to be defined), we opted to reuse the filesystem to provide access control by attaching LITMUS $^{\mathrm{RT}}$ resources at run-time to inodes (an inode is the in-kernel representation of a file). When a task attempts to obtain a reference to a resource, it specifies a file descriptor to be used as the naming context. By specifying the same file, synchronization across process boundaries is possible (but only if allowed by the appropriate permissions). If permitted, the kernel locates the requested resource and stores its address in a lookup table in the thread control block (TCB). Similar to the concept of the file descriptor table, the resource lookup table enables fast referenceto-address translation in the performance critical path of synchronization-related system calls. With the new method, LITMUS $^{\mathrm{RT}}$ resources are created dynamically on demand.

Priority ceilings. It is commonly claimed that protocols such as the PCP are hard to use in practice because priority ceilings must be determined offline and specified manually at runtime. However, that is not the case, as ceilings can be computed automatically when threads obtain references to resources.

The priority ceiling of a resource is initially $\infty$ (INT_MAX in practice) and raised (if necessary) when a realtime task obtains a reference to it. To ensure correctness, no thread may request a resource before all tasks that share the resource have obtained a reference (for that resource). Otherwise, the computed ceiling may be incorrect. In practice, this problem does not occur since it is ensured that the initialization of all real-time tasks is complete by the time the first job of any task is released.

A processor's system ceiling is maintained as a stack of the local resources that are currently in use. Under the SRP, when a task releases a new job or a job resumes, the kernel checks whether the task's priority exceeds the priority ceiling of the top-most resource on the system ceiling stack (unless the stack is empty). If the job's priority does not exceed the ceiling, then it is added to a per-processor wait queue (a wait queue is a standard Linux component used to suspend jobs; see below). When an SRP resource is popped off the system ceiling stack, jobs with priorities exceeding the new system ceiling are resumed. Under the PCP, the top-most resource's priority ceiling is checked every time a resource is requested.

In our experience, automatic determination of priority ceilings facilitates task system setup greatly and eliminates the possibility for human error.
Priority inheritance. Transitive priority inheritance, as mandated by the PCP, requires the kernel to be able to traverse the "wait-for" dependency graph to arbitrary depths. The necessary state information is kept partially in the TCBs and partially in the resource objects. When a thread is blocked, the address of the resource is stored in its TCB. Similarly, the address of the holding thread is stored in the resource object.

When a job $T_{i}^{j}$ blocks on a PCP resource $\ell$ held by $T_{k}^{l}$ (as determined by the address stored in the resource object) and $T_{i}^{j}$ has a higher effective priority than $T_{k}^{l}$, then $T_{k}^{l}$ 's TCB is updated to reflect that it inherits $T_{i}^{j}$ 's effective priority. If $T_{k}^{l}$ is already blocked on another PCP resource (as indicated by its TCB), then transitive priority inheritance is triggered. $T_{k}^{l}$,s effective priority is recomputed when it releases $\ell$ by examining all PCP resources that $T_{k}^{l}$ holds at the time of release.

Wait queues. In Linux, threads suspend by enqueuing themselves in a wait queue, which is a reusable component used throughout the kernel. However, the standard Linux API does not enforce any ordering of blocked threads. Modifications were required to ensure strict ordering under the FMLP (FIFO order), and the M-PCP and D-PCP (priority order).

Under the PCP, each resource has its own wait queue to control priority inheritance. (The SRP only requires a single wait queue per processor). When a PCP resource is released, all jobs in its wait queue are resumed - staticpriority scheduling ensures that the highest-priority blocked job will proceed next. This has the great benefit that the PCP does not actually require sorted priority queues.

Sha et al. [27] and Rajkumar [25] note that an implementation of the PCP does not necessarily require per-resource wait queues. Instead, they propose to keep blocked jobs in the ready queue since the priority order will ensure that they do not execute prematurely. This may be a valid approach for an OS in a closely controlled setting (e.g., in embedded systems), but for a general purpose OS such as Linux, it is not a sufficiently robust approach. This is because it relies on correct behavior on the part of resource-holding jobs. What happens if resource-holding jobs suspend unexpectedly? If blocked tasks are kept on the run queue, such an event would allow two or more jobs to execute in a critical section - a behavior that is clearly not correct. One might argue that in a correct real-time system the resourceholding job does not block. However, in real-world systems such behavior cannot be ruled out. Even a simple printf statement, maybe inserted for debugging purposes, can lead to (very short) suspensions. Similarly, an unexpected page fault due to the omission of disabling demand paging might also cause a lock-holding task to suspend. Again, such an event will not occur in a correct real-time system, but cannot be ruled out completely (especially during development). In 
the interest of robustness, a kernel-based mutual-exclusion primitive should not rely on the correctness of user-space programs. Instead, it should react as gracefully as possible when facing incorrect applications.

Atomicity of resource requests. Since the FMLP requires jobs holding a long resource to be non-preemptable (under partitioned scheduling), care must be taken to ensure that group lock acquisition and non-preemptivity are enacted atomically, i.e., if a job were to enter its critical section in a second step, then it could be preempted in the time between these two events. The LITMUS ${ }^{\mathrm{RT}}$ kernel avoids this race condition by marking the resource-holding thread as non-preemptable before returning to user space.

D-PCP. Due to the use of local agents, the D-PCP implementation differs significantly from the M-PCP and FMLP implementations. There are two approaches for realizing the concept of a local agent: (i) since LITMUS ${ }^{\mathrm{RT}}$ supports exclusively shared-memory architectures, the requesting thread could be migrated to the processor where the resource resides; or (ii) an additional thread is provided to serve as the local agent. Since we conjecture that losing cache affinity due to a migration is more expensive than sending a request, we chose to implement approach (ii) in LITMUS $^{\mathrm{RT}}$. Note that, since only one local agent can execute at any time, providing a local agent thread for each remote task is unnecessary - it suffices to provide one local agent thread per address space that contains global resources. In practice, we provide a local agent for each resource anyway - assuming that every resource resides in its own address space is always correct and simplifies the implementation significantly.

Performance comparison. Due to space constraints, we are unable to thoroughly compare the implemented synchronization approaches. A detailed study incorporating real-word overheads is currently in preparation [7].

However, to give a rough estimate of relative performance, Table 1 shows average and maximum observed system call overheads, which were recorded on a system consisting of four Intel Xeon processors clocked at $2.7 \mathrm{GHz}$. For each protocol, we measured the request and release overhead based on over 300,000 pairs of timestamps that were recorded just before and after the system calls of interest. The worst-case and average overheads were determined after discarding the top one percent of data points to filter for interrupts and other noise (similar to the methodology used in [10]). Note that the system was mostly idle during these measurements. The obtained values thus are only meaningful relative to each other, but do not necessarily reflect a worst-case scenario. We are currently engaged in experiments to obtain more realistic worst-case overheads [7].

Based on these results, we conclude that local synchronization protocols are slightly more efficient to implement

\begin{tabular}{|l|r|r|}
\hline Protocol & Request & Release \\
\hline SRP & $0.36(0.56)$ & $0.43(0.50)$ \\
PCP & $0.38(0.49)$ & $0.46(0.52)$ \\
M-PCP & $0.58(0.66)$ & $0.52(0.59)$ \\
D-PCP & $6.91(8.08)$ & $5.91(6.57)$ \\
FMLP (long) & $0.51(0.56)$ & $0.59(0.61)$ \\
FMLP (short) & $0.19(0.21)$ & $0.09(0.09)$ \\
\hline
\end{tabular}

Table 1: Average (maximum) overheads encountered for invoking kernel-based synchronization protocols. All times are in $\mu$ s.

than suspension-based global shared-memory synchronization protocols. Of great interest are the costs associated with the D-PCP. Due to its distributed nature (which requires IPC), its overhead is an order of magnitude larger than that of shared-memory global synchronization protocols. This discrepancy makes it unlikely that the D-PCP is a favorable choice for synchronization on shared-memory multiprocessors. However, more detailed studies are required to obtain a definitive answer.

\section{Conclusion}

In this paper, we have extended the FMLP to P-SP scheduling and bounded its worst-case blocking behavior (in the online version of the paper). Further, we have presented the first implementation that integrates the SRP, the PCP, the M-PCP, the D-PCP, and the FMLP in a single framework in a general-purpose OS. We also discussed some of the architectural design issues that arise when implementing real-time synchronization protocols in such an OS. We are currently preparing an extensive performance comparison of the aforementioned synchronization protocols, which will be presented in a companion paper to this work [7].

Lessons learned. In our ongoing work with Linux and LITMUS $^{\mathrm{RT}}$ in particular, we have come to recognize three principles that were not readily apparent to us prior to our implementation efforts.

1. Robustness is essential. Algorithms that produce mostly correct results when faced with small "glitches" are always preferable to algorithms that have superior theoretical performance but fail catastrophically when assumptions are violated. In practice, it is impossible to foresee all possible interactions in a complex general-purpose OS such as Linux.

2. Algorithmic performance dominates. On our platform, the impact of non-determinism inherent in Linux (such as interrupt handlers) is small compared to the impact that real-time algorithms have on determinism - interrupts rarely execute for longer than $100 \mu \mathrm{s}$. In contrast, even a single-quantum priority-inversion will delay a thread by (at least) $1 \mathrm{~ms}$ (which is the quantum size in many variants of Linux). Thus, for the vast majority 
of time-sensitive applications that do not require submillisecond response times, a lack of proper real-time scheduling and synchronization support has far greater consequences than other sources of OS latency.

3. Design for change. Linux is a fast-moving target. The rate of change can be overwhelming for an academic research group. When implementing prototypes in Linux, always choose the least-intrusive implementation possible. In our experience, architectures that are structured as a layer of patches work best.

Several interesting avenues for the future present themselves. While the FMLP now supports several major multiprocessor scheduling algorithms, it would be beneficial to extend the FMLP to PD $^{2}$ [2], Earliest-Deadline-until-ZeroLaxity [23], and utility-based [18] scheduling. Finally, we would like to analyze the impact of multicore architectures on the performance of real-time resoure sharing algorithms.

\section{References}

[1] IBM and Red Hat announce new development innovations in Linux kernel. Press release. http://www-03.ibm.com/ press/us/en/pressrelease/21232.wss, 2007.

[2] J. Anderson and A. Srinivasan. Mixed Pfair/ERfair scheduling of asynchronous periodic tasks. Journal of Computer and System Sciences, 68(1):157-204, 2004.

[3] T. Anderson. The performance of spin lock alternatives for shared-memory multiprocessors. IEEE Transactions on Parallel and Distributed Systems, 1(1):6-16, 1990.

[4] T. Baker. Stack-based scheduling of real-time processes. Journal of Real-Time systems, 3(1):67-99, 1991.

[5] S. Bisson. Azul announces 192 core Java appliance. http://www.itpro.co.uk/serves/news/99765/azul-announces1 92-core-java-appliance.html, 2006.

[6] A. Block, H. Leontyev, B. Brandenburg, and J. Anderson. A flexible real-time locking protocol for multiprocessors. In Proceedings of the 13th IEEE International Conference on Embedded and Real-Time Computing Systems and Applications, pages 71-80, 2007.

[7] B. Brandenburg and J. Anderson. A comparison of the MPCP, D-PCP and the FMLP on LITMUS ${ }^{\mathrm{RT}}$. In submission.

[8] B. Brandenburg and J. Anderson. Feather trace: A lightweight event tracing toolkit. In Proceedings of the Third International Workshop on Operating Systems Platforms for Embedded Real-Time Applications, pages 19-28, 2007.

[9] B. Brandenburg, A. Block, J. Calandrino, U. Devi, H. Leontyev, and J. Anderson. LITMUS ${ }^{\mathrm{RT}}$ : A status report. In Proceedings of the 9th Real-Time Workshop, pages 107-123. Real-Time Linux Foundation, 2007.

[10] B. Brandenburg, J. Calandrino, A. Block, H. Leontyev, and J. Anderson. Synchronization on real-time multiprocessors: To block or not to block, t o suspend or spin? In Proceedings of the 14th IEEE Real-Time and Embedded Technology and Applications Symposium, pages 342-353, 2008.
[11] B. Brandenburg and J.Anderson. An implementation of the PCP, SRP, D-PCP, M-PCP, and FMLP real-time synchronization protocols in LITMUS ${ }^{\mathrm{RT}}$ (extended version). http://www.cs.unc.edu/ anderson/papers.html.

[12] J. Calandrino, J. Anderson, and D. Baumberger. A hybrid real-time scheduling approach for large-scale multicore platforms. In Proceedings of the 19th Euromicro Conference on Real-Time Systems, pages 247-256, 2007.

[13] J. Calandrino, H., A. Block, U. Devi, and J. Anderson. LITMUS $^{\mathrm{RT}}$ : A testbed for empirically comparing real-time multiprocessor schedulers. In Proceedings of the 27th IEEE Real-Time Systems Symposium, pages 111-123, 2006.

[14] Chia-Mei Chen and Satish K. Tripathi. Multiprocessor priority ceiling based protocols. Technical Report CR-TR-3252, University of Maryland, 1994.

[15] U. Devi. Soft Real-Time Scheduling on Multiprocessors. PhD thesis, University of North Carolina, Chapel Hill, NC, 2006.

[16] P. Gai, M. di Natale, G. Lipari, A. Ferrari, C.Gabellini, and P. Marceca. A comparison of MPCP and MSRP when sharing resources in the Janus multiple processor on a chip platform. In Proceedings of the 9th IEEE Real-Time And Embedded Technology Application Symposium, pages 189-198, 2003.

[17] UNC Real-Time Group. LITMUS ${ }^{\mathrm{RT}}$ homepage. http://www.cs.unc.edu/ anderson/litmus-rt.

[18] E. Jensen, C. Locke, and H. Tokuda. A time-driven scheduling model for real-time systems. In 6th IEEE Real-Time Systems Symposium, pages 112-122, 1985.

[19] C. Liu and J. Layland. Scheduling algorithms for multiprogramming in a hard real-time environment. Journal of the ACM, 30:46-61, 1973.

[20] J.W.S. Liu. Real-Time Systems. Prentice Hall, 2000.

[21] J. M. López, J. L. Díaz, and D. F. García. Utilization bounds for edf scheduling on real-time multiprocessor systems. Real-Time Systems, 28(1):39-68, 2004.

[22] SUN Microsystems. SUN UltraSPARC T1. Marketing material. http://www.sun.com/ processors/UltraSPARC-T1/, 2008.

[23] X. Piao, S. Han, H. Kim, M. Park, Y. Cho, and S. Cho. Predictability of earliest deadline zero laxity algorithm for multiprocessor real-time systems. 9th International Symposium on Object and Component-Oriented Real-Time Distributed Computing, 2006.

[24] R. Rajkumar. Real-time synchronization protocols for shared memory multiprocessors. 10th International Conference on Distributed Computing Systems, pages 116-123, 1990.

[25] R. Rajkumar. Synchronization In Real-Time Systems - A Priority Inheritance Approach. Kluwer Academic Publishers, 1991.

[26] R. Rajkumar, L. Sha, and J.P. Lehoczky. Real-time synchronization protocols for multiprocessors. Real-Time Systems Symposium, 1988., Proceedings., pages 259-269, 1988.

[27] L. Sha, R. Rajkumar, and J. P. Lehoczky. Priority inheritance protocols: An approach to real-time synchronization. IEEE Transactions on Computers, 39(9):1175-1185, 1990.

[28] S. Shankland and M. Kanellos. Intel to elaborate on new multicore processor. http://news.zdnet.co.uk/hardware/ chips/0,39020354,39116043,00.htm, 2003. 


\section{A Bounding Blocking Time}

Blocking is any delay encountered by a job that would not have arisen if all tasks were independent. The maximum blocking duration must be bounded and accounted for when testing schedulability. In this appendix, we derive a bound $\mathrm{B}\left(T_{i}^{j}\right)$ for the worst-case blocking incurred by any job $T_{i}^{j}$ under the FMLP under P-SP scheduling. Under the FMLP, there are five distinct sources of blocking that can delay a job $T_{i}^{j}$ :

1. Boost blocking, bounded by $\mathrm{BB}\left(T_{i}^{j}\right)$, is incurred when $T_{i}^{j}$ cannot be scheduled because a local lower-priority job is executing a long request. If none of the lower-priority tasks on $T_{i}^{j}$,s processor share long resources, then $\mathrm{BB}\left(T_{i}^{j}\right)=0$.

2. Arrival blocking, bounded by $\mathrm{AB}\left(T_{i}^{j}\right)$, is incurred when $T_{i}^{j}$ becomes eligible for execution (either on release or when resuming from a suspension) and cannot be scheduled because the currently scheduled job executes non-preemptively. Note that only outermost short requests that are not nested within a long request are considered to cause arrival blocking since $\mathrm{BB}\left(T_{i}^{j}\right)$ includes short requests nested within long requests. If none of the lower-priority tasks on $T_{i}^{j}$,s processor share short resources, then $\mathrm{AB}\left(T_{i}^{j}\right)=0$.

3. Short blocking, bounded by $\mathrm{SB}\left(T_{i}^{j}\right)$, is incurred while a request of $T_{i}^{j}$ for a short resource $\ell$ cannot be satisfied because another remote job currently holds $\ell$ 's group lock. Local jobs cannot cause direct short blocking since the whole request is executed non-preemptively, i.e., short requests are atomic with regard to local jobs. If $T_{i}^{j}$ does not share short resources, then $\mathrm{SB}\left(T_{i}^{j}\right)=0$.

4. Long blocking, bounded by $\operatorname{LB}\left(T_{i}^{j}\right)$, is incurred while a request of $T_{i}^{j}$ for a long resource $\ell$ cannot be satisfied because another remote job currently holds $\ell$ 's group lock. Only remote jobs are considered to cause long blocking-local higher-priority jobs do not block $T_{i}^{j}$ (since they would have had precedence even without resource sharing), and any delay due to long requests by lower-priority jobs is already accounted for in $\operatorname{BB}\left(T_{i}^{j}\right)$. If $T_{i}^{j}$ does not share long resources, then $\operatorname{LB}\left(T_{i}^{j}\right)=0$.

5. Deferral blocking, bounded by $\mathrm{DB}\left(T_{i}\right)$, is incurred when higher-priority a job defers some of its execution to a later instant (due to suspensions) and then causes a job of $T_{i}$ to experience increased competition for processor time. (Deferred execution was studied thoroughly in the context of deferrable servers and is explained well in [20].) If none of the higher-priority tasks on $T_{i}^{j}$, s processor share long resources, then $\mathrm{DB}\left(T_{i}\right)=0$.

Given bounds for these sources of blocking, a bound for the worst-case blocking of $T_{i}^{j}$ is given by

$$
\mathrm{B}\left(T_{i}^{j}\right)=\mathrm{BB}\left(T_{i}^{j}\right)+\mathrm{AB}\left(T_{i}^{j}\right)+\mathrm{SB}\left(T_{i}^{j}\right)+\mathrm{LB}\left(T_{i}^{j}\right)+\mathrm{DB}\left(T_{i}\right)
$$

Approach. Boost blocking, arrival blocking, and deferral blocking mostly depend on the behavior of jobs other than $T_{i}^{j}$ - they can be bounded by determining the worst-case request pattern created by local jobs. Short blocking and long blocking depend on the behavior of both $T_{i}^{j}$ and remote jobs. Intuitively, blocking incurred by $T_{i}^{j}$ due to resource requests issued by $T_{i}^{j}$ can be bounded by the minimum of the number of times that $T_{i}^{j}$ requests resources and he number of times that remote jobs request shared resources-in order for interference to occur, both conflicting parties must have issued a request. Note that this is a direct consequence of using FIFO queuing: if $T_{i}^{j}$ makes only one request for a resource $\ell$, then it can be directly blocked at most once by remote task that accesses resources in $\mathrm{G}(\ell)$ no matter how many conflicting requests are issued while $T_{i}^{j}$ is pending. In contrast, if wait queues are ordered by priority (as is it is the case under the M-PCP and the D-PCP), then a single request by $T_{i}^{j}$ may lead to $T_{i}^{j}$ being blocked multiple times by each remote higher-priority task.

In the following subsections, we summarize the notation used throughout this appendix and derive the individual bounds based on the intuition described above. 


\section{A.1 Notation}

For convenience, some of the earlier definitions are repeated.

$T_{i} \quad$ A task with priority $i$ (priorities are assumed to be unique).

$T_{i}^{j} \quad$ The $j$ th job of $T_{i}$.

jobs $\left(T_{i}\right) \quad$ The set of all jobs released by $T_{i}$ (may be infinite).

$\mathrm{e}\left(T_{i}\right) \quad$ The worst-case execution cost of $T_{i}$.

$\mathrm{p}\left(T_{i}\right) \quad$ The period of $T_{i}$.

$\mathrm{P}\left(T_{i}\right) \quad$ The processor $(1 \ldots m)$ to which $T_{i}$ is assigned.

$\ell \quad$ A resource (either short or long).

$\mathcal{R} \quad$ A request for a resource (also either short or long).

$\operatorname{subreq}(\mathcal{R}) \quad$ Predicate; true iff $\mathcal{R}$ is short outermost but nested within a long request $\mathcal{R}^{\prime}$, i.e., $\mathcal{R}^{\prime}$ contains $\mathcal{R}$.

$\operatorname{res}(\mathcal{R}) \quad$ The resource requested by $\mathcal{R}$.

$\operatorname{tsk}(\mathcal{R}) \quad$ The task that issues $\mathcal{R}$.

$|\mathcal{R}| \quad$ An upper bound on the maximum duration for which $\operatorname{res}(\mathcal{R})$ will be held.

Does not include blocking due to $\mathcal{R}$. Includes blocking due to nested requests.

$\mathrm{G}(\ell) \quad$ The resource group that contains $\ell$.

$\operatorname{SR}\left(T_{i}^{j}\right) \quad$ The set of outermost short requests issued by $T_{i}^{j}$.

$\operatorname{LR}\left(T_{i}^{j}\right) \quad$ The set of outermost long requests issued by $T_{i}^{j}$.

$\operatorname{WCSR}\left(T_{i}\right) \quad$ A set of outermost short requests that bounds the worst-case behavior of any job of $T_{i}$.

(W.r.t. duration and number of requests; used when the exact identity of an interfering job is unknown.)

$\operatorname{WCLR}\left(T_{i}\right) \quad$ A set of outermost long requests that bounds the worst-case behavior of any job of $T_{i}$.

(W.r.t. duration and number of requests; used when the exact identity of an interfering job is unknown.)

There are $n$ tasks, ordered by priority. The highest priority is $i=1$, the lowest priority is $i=n$. For notational convenience, we assume $\max (\emptyset)=0$.

\section{A.2 Basic Definitions}

In this subsection, we define terms for recurring concepts in the derivation of the blocking terms.

The set of tasks assigned to processor $p$ is given by

$$
\operatorname{partition}(p)=\left\{T_{x} \mid \mathrm{P}\left(T_{x}\right)=p\right\} \text {. }
$$

Given a request $\mathcal{R}$, the set of requests issued by jobs on processor $p$ that can interfere with $\mathcal{R}$ is given by

$$
\operatorname{competing}(\mathcal{R}, p)=\left\{\mathcal{R}^{\prime} \mid \mathrm{G}(\operatorname{res}(\mathcal{R}))=\mathrm{G}\left(\operatorname{res}\left(\mathcal{R}^{\prime}\right)\right) \wedge t \operatorname{sk}(\mathcal{R}) \neq \operatorname{tsk}\left(\mathcal{R}^{\prime}\right) \wedge \mathrm{P}\left(\operatorname{tsk}\left(\mathcal{R}^{\prime}\right)\right)=p\right\} .
$$

Given a job $T_{i}^{j}$ and a task $T_{x}$, we let $w \operatorname{cl} x\left(T_{x}, T_{i}^{j}\right)$ denote the worst-case set of long outermost requests issued by jobs of $T_{x}$ while $T_{i}^{j}$ is pending. Since multiple jobs of $T_{x}$ can block $T_{i}^{j}$, it is important to keep track of the multiple instances of each request that $T_{i}^{j}$ can encounter- this is realized by tagging competing requests in the definition of the set. There are at most $\left\lceil\frac{\mathbf{p}\left(T_{i}\right)}{\mathbf{p}\left(T_{x}\right)}\right\rceil+1$ jobs of $T_{x}$ concurrently active with $T_{i}^{j}$. Hence, the set is given by

$$
w \operatorname{cl}\left(T_{x}, T_{i}^{j}\right)=\left\{(\mathcal{R}, k) \mid \mathcal{R} \in \operatorname{WCLR}\left(T_{x}\right) \wedge k \in\left\{1, \ldots,\left\lceil\frac{\mathrm{p}\left(T_{i}\right)}{\mathrm{p}\left(T_{x}\right)}\right\rceil+1\right\}\right\} .
$$

Similarly, we define for outermost short requests that are not nested within a long request

$$
\operatorname{wcs} x\left(T_{x}, T_{i}^{j}\right)=\left\{(\mathcal{R}, k) \mid \mathcal{R} \in \operatorname{WCSR}\left(T_{x}\right) \wedge \neg \operatorname{subreq}(\mathcal{R}) \wedge k \in\left\{1, \ldots,\left\lceil\frac{\mathrm{p}\left(T_{i}\right)}{\mathrm{p}\left(T_{x}\right)}\right\rceil+1\right\}\right\} .
$$


We let $w c l x^{>}\left(T_{x}, T_{i}^{j}\right)$ denote the set $w c l x\left(T_{x}, T_{i}^{j}\right)$ ordered by non-decreasing request duration and let $w c l x_{l}^{>}\left(T_{x}, T_{i}^{j}\right)$ denote the $l$ th item in $w c l x^{>}\left(T_{x}, T_{i}^{j}\right)$ (if it exists), i.e., $w c l x^{>}\left(T_{x}, T_{i}^{j}\right)$ is the list obtained by sorting $w c l x\left(T_{x}, T_{i}^{j}\right)$ with the following relation:

$$
(\mathcal{R}, k)>\left(\mathcal{R}^{\prime}, k^{\prime}\right) \Leftrightarrow|\mathcal{R}|>\left|\mathcal{R}^{\prime}\right|
$$

When a job becomes eligible to execute (either on release or when resuming execution after a suspension) it is said to arrive. An upper bound on the number of arrivals of a job $T_{i}^{j}$ is given by

$$
\operatorname{narr}\left(T_{i}^{j}\right)=1+\left|\operatorname{LR}\left(T_{i}^{j}\right)\right|
$$

\section{A.3 Bounding Boost Blocking: $\mathrm{BB}\left(T_{i}^{j}\right)$}

Boost blocking occurs when a lower-priority job has its priority boosted and thereby prevents $T_{i}^{j}$ from being scheduled. Since lower-priority jobs cannot issue requests while $T_{i}^{j}$ is eligible for execution (they are not scheduled), requests that cause boost blocking must have been made either before $T_{i}^{j}$ was released or while $T_{i}^{j}$ was suspended. Thus, a lowerpriority task $T_{x}$ can cause boost blocking at most once every time $T_{i}^{j}$ arrives. Hence, the duration of boost blocking caused by $T_{x}$ is bounded by the sum of the durations of the $\operatorname{narr}\left(T_{i}^{j}\right)$ longest requests (for long resources) issued by jobs of $T_{x}$ while $T_{i}^{j}$ is pending. Let $a=\operatorname{narr}\left(T_{i}^{j}\right), s=\left|w c l x\left(T_{x}, T_{i}^{j}\right)\right|$, and $\left(\mathcal{R}_{l}, k_{l}\right)=w c l x_{l}^{>}\left(T_{x}, T_{i}^{j}\right)$. Then

$$
\operatorname{bbt}\left(T_{x}, T_{i}^{j}\right)=\sum_{l=1}^{\min (a, s)}\left|\mathcal{R}_{l}\right| .
$$

A bound for the maximum duration of boost-blocking incurred by $T_{i}^{j}$ is then given by

$$
\mathrm{BB}\left(T_{i}^{j}\right)=\sum_{\substack{x>i \\ \mathrm{P}\left(T_{x}\right)=\mathrm{P}\left(T_{i}\right)}} b b t\left(T_{x}, T_{i}^{j}\right) .
$$

\section{A.4 Bounding Arrival Blocking: $\mathrm{AB}\left(T_{i}^{j}\right)$}

$\mathrm{BB}\left(T_{i}^{j}\right)$ accounts for most blocking due to non-preemptive sections. However, every time $T_{i}^{j}$ arrives and becomes eligible for execution, it can be blocked by at most one local lower-priority job executing non-preemptively a short request that is not nested within a long request. The set of all short requests that can block $T_{i}^{j}$ on arrival is given by

$$
\operatorname{abr}\left(T_{i}^{j}\right)=\bigcup_{\substack{\mathrm{P}\left(T_{x}\right)=\mathrm{P}\left(T_{i}\right) \\ x>i}} w \operatorname{cs} x\left(T_{x}, T_{i}^{j}\right) .
$$

However, $T_{i}^{j}$ can be blocked on arrival at most $\operatorname{narr}\left(T_{i}^{j}\right)$ times. Let $a b r^{>}\left(T_{i}^{j}\right)$ denote the set $a b r\left(T_{i}^{j}\right)$ ordered by nondecreasing request duration and let $a b r_{l}^{>}\left(T_{i}^{j}\right)$ denote the lth item in $a b r^{>}\left(T_{i}^{j}\right)$ (akin to $w c l x^{>}\left(T_{x}, T_{i}^{j}\right)$ ). Given this list, a bound on arrival blocking is given by

$$
\mathrm{AB}\left(T_{i}^{j}\right)=\sum_{l=1}^{\min (a, s)}\left|\mathcal{R}_{l}\right|
$$

where $a=\operatorname{narr}\left(T_{i}^{j}\right), s=\left|\operatorname{abr}\left(T_{i}^{j}\right)\right|$, and $\left(\mathcal{R}_{l}, k_{l}\right)=a b r_{l}^{>}\left(T_{i}^{j}\right)$. 


\section{A.5 Bounding Short Blocking: $\mathrm{SB}\left(T_{i}^{j}\right)$}

In the worst case, $T_{i}^{j}$ is the last job to enter the spin queue on every short request it makes. However, due to nonpreemptivity, there is at most one job on at most $m-1$ other processors ahead of $T_{i}^{j}$ in the spin queue. Thus, the per-request blocking can be bounded by the sum of the durations of the longest potentially competing request on each other processor.

$$
\operatorname{sbr}(\mathcal{R})=\sum_{\substack{p=1 \\ p \neq \mathrm{P}(\operatorname{tsk}(\mathcal{R}))}}^{m} \max \left(\left\{\left|\mathcal{R}_{c}\right| \mid \mathcal{R}_{c} \in \operatorname{competing}(\mathcal{R}, p)\right\}\right)
$$

Overall short blocking is bounded by the sum of the worst-case blocking incurred from each short request made by $T_{i}^{j}$ :

$$
\mathrm{SB}\left(T_{i}^{j}\right)=\sum_{\mathcal{R} \in \mathrm{SR}\left(T_{i}^{j}\right)} \operatorname{sbr}(\mathcal{R})
$$

\section{A.6 Bounding Long Blocking: $\operatorname{LB}\left(T_{i}^{j}\right)$}

Every time $T_{i}^{j}$ requests a long resource, it can be subject to three ways of blocking.

1. Direct remote blocking occurs when a remote job that is executing holds the group lock for the resource that $T_{i}^{j}$ requested.

2. Transitive remote boost blocking occurs when $T_{i}^{j}$ is directly blocked by a remote job $T_{r}^{k}$, and $T_{r}^{k}$ is not scheduled because other priority-boosted jobs take precedence over $T_{r}^{k}$ (recall that priority-boosted jobs are scheduled in FIFO order). Note that $T_{i}^{j}$ can incur transitive remote boost blocking even when $T_{r}^{k}$ is not experiencing boost blocking itself: higher-priority jobs on $T_{r}^{k}$, s processor never block $T_{r}^{k}$, but they do block $T_{i}^{j}$ if they execute while $T_{r}^{k}$ holds the group lock that $T_{i}^{j}$ requires to proceed (since, without resource sharing, $T_{i}^{j}$ would have proceeded unhindered). If transitive remote boost blocking takes place, then it precedes direct remote blocking.

3. Transitive remote arrival blocking occurs when $T_{i}^{j}$ is directly blocked by a remote job $T_{r}^{k}$, and $T_{r}^{k}$ cannot be scheduled upon resuming because another job $T_{n p}^{l}$ on $T_{r}^{k}$ 's processor is non-preemptively executing a short request. Note that at most one non-preemptive section can block $T_{r}^{k}$, that only short outermost requests not nested within long requests a considered to cause transitive remote arrival blocking (since nested outermost short requests are part of transitive remote boost blocking), and that $T_{i}^{j}$ incurs transitive remote arrival blocking even when $k>n p$.

Items 1 and 2 taken together imply that every time $T_{i}^{j}$ is directly blocked by a job on a remote processor $p$ it can be blocked by one long request (for any resource) by each job on processor $p$. Item 3 implies that every time $T_{i}^{j}$ is directly blocked by a job on a remote processor $p$ it can be blocked by one short request (for any resource) by one job on processor $p$. We let $r b l\left(T_{i}^{j}\right)$ (rbl: remote blocking - long) denote a bound on the former and let $r b s\left(T_{i}^{j}\right)$ (rbs: remote blocking - short) denote a bound on the latter. Given these, a bound on blocking due to long requests incurred by $T_{i}^{j}$ is given by

$$
\mathrm{LB}\left(T_{i}^{j}\right)=\operatorname{rbs}\left(T_{i}^{j}\right)+\operatorname{rbl}\left(T_{i}^{j}\right)
$$

Bounding the number of times $T_{i}^{j}$ is directly blocked on a processor $p$ due to long requests: $n d b p\left(T_{i}^{j}, p\right)$. We first derive a bound for the number of times that $T_{i}^{j}$ can be directly blocked on each processor since both $r b l\left(T_{i}^{j}\right)$ and $r b s\left(T_{i}^{j}\right)$ depend on it. We can bound $n d b p\left(T_{i}^{j}, p\right)$ by the sum of times that jobs from each task $T_{x}$ on $p$ can directly block $T_{i}^{j}$ (denoted $\operatorname{ndbt}\left(T_{x}, T_{i}^{j}\right)$ )

$$
n d b p\left(T_{i}^{j}, p\right)=\sum_{T_{x} \in \operatorname{partition}(p)} \operatorname{ndbt}\left(T_{x}, T_{i}^{j}\right),
$$


which in turn can be bounded by the sum of the times that jobs from $T_{x}$ can block $T_{i}^{j}$ via each group $g$ that $T_{i}^{j}$ accesses (denoted $\operatorname{ndbtg}\left(T_{x}, T_{i}^{j}, g\right)$ )

$$
\operatorname{ndbt}\left(T_{x}, T_{i}^{j}\right)=\sum_{g \in\left\{\mathrm{G}(\mathcal{R}) \mid \mathcal{R} \in \operatorname{LR}\left(T_{i}^{j}\right)\right\}} \operatorname{ndbtg}\left(T_{x}, T_{i}^{j}, g\right) .
$$

A bound on the number of times that jobs of $T_{x}$ interfere with $T_{i}^{j}$ via group $g$ is simply the minimum of the times that either $T_{i}^{j}$ or jobs of $T_{x}$ acquire $g$ 's group lock while $T_{i}^{j}$ is pending. Note that a pessimistic assumption on the number of jobs of $T_{x}$ that are competing with $T_{i}^{j}$ must be made.

$$
\operatorname{ndbtg}\left(T_{x}, T_{i}^{j}, g\right)=\min \left(\begin{array}{c}
\left|\left\{\mathcal{R} \mid \mathcal{R} \in \mathrm{LR}\left(T_{i}^{j}\right) \wedge \mathrm{G}(\mathcal{R})=g\right\}\right|, \\
\left|\left\{\mathcal{R} \mid \mathcal{R} \in \operatorname{WCLR}\left(T_{x}\right) \wedge \mathrm{G}(\mathcal{R})=g\right\}\right| \cdot\left(\left\lceil\frac{\mathrm{p}\left(T_{i}\right)}{\mathrm{p}\left(T_{x}\right)}\right\rceil+1\right)
\end{array}\right)
$$

Bounding remote blocking - long: $\operatorname{rbl}\left(T_{i}^{j}\right)$. A bound for $r b l\left(T_{i}^{j}\right)$ can be obtained by analyzing the interference from each remote processor independently since jobs blocking $T_{i}^{j}$ are not subject to interference from other processors while they block $T_{i}^{j}$. Hence, given a bound on the total interference from a processor $p$ (denoted $r b l p\left(T_{i}^{j}, p\right)$ ), a bound for $r b l\left(T_{i}^{j}\right)$ is given by

$$
\operatorname{rbl}\left(T_{i}^{j}\right)=\sum_{\substack{p=1 \\ p \neq \mathrm{P}\left(T_{i}\right)}}^{m} \operatorname{rblp}\left(T_{i}^{j}, p\right) .
$$

A bound on the interference from processor $p$ is simply the sum of the bounds on the interference from each task on $p$; formally

$$
\operatorname{rblp}\left(T_{i}^{j}, p\right)=\sum_{T_{x} \in \operatorname{partition}(p)} \operatorname{rblt}\left(T_{x}, T_{i}^{j}\right) .
$$

Jobs of $T_{x}$ can block $T_{i}^{j}$ at most once every time $T_{i}^{j}$ is blocked on $T_{x}$ 's processor. Hence, interference by $T_{x}$ can be bounded by the sum of the durations of the top $n d b p\left(T_{i}^{j}, \mathrm{P}\left(T_{x}\right)\right)$ longest requests for long resources issued by jobs of $T_{x}$ while $T_{i}^{j}$ is pending. Formally, let $s=\left|w c l x\left(T_{x}, T_{i}^{j}\right)\right|, b=n d b p\left(T_{i}^{j}, \mathrm{P}\left(T_{x}\right)\right)$ and $\left(\mathcal{R}_{l}, k_{l}\right)=w c l x_{l}^{>}\left(T_{x}, T_{i}^{j}\right)$, then

$$
\operatorname{rblt}\left(T_{x}, T_{i}^{j}\right)=\sum_{l=1}^{\min (b, s)}\left|\mathcal{R}_{l}\right| .
$$

Bounding remote blocking - short: $\operatorname{rbs}\left(T_{i}^{j}\right)$. Similar to the $r b l\left(T_{i}^{j}\right)$ case, $r b s\left(T_{i}^{j}\right)$ can be analyzed per processor. Hence a bound is given by

$$
\operatorname{rbs}\left(T_{i}^{j}\right)=\sum_{\substack{p=1 \\ p \neq \mathrm{P}\left(T_{i}\right)}}^{m} \operatorname{rbsp}\left(T_{i}^{j}, p\right) .
$$

Recall that $T_{i}^{j}$ can incur transitive blocking from one short request every time that it is directly blocked (when requesting a long resource) by a remote job. We let $w \operatorname{csp}\left(T_{i}^{j}, p\right)$ denote the set of all short requests that can cause $T_{i}^{j}$ transitive remote arrival blocking:

$$
w c s p\left(T_{i}^{j}, p\right)=\bigcup_{T_{x} \in \operatorname{partition}(p)} \operatorname{wcs} x\left(T_{x}, T_{i}^{j}\right)
$$


The rbs interference caused by jobs on processor $p$ can be bounded by the sum of the durations of the top $n d b p\left(T_{i}^{j}, p\right)$ requests in $w \operatorname{csp}\left(T_{i}^{j}, p\right)$. Formally, let $w \operatorname{csp} p_{l}^{>}\left(T_{x}, T_{i}^{j}\right)$ denote the $l$ th element in the ordered set $w c s p^{>}\left(T_{i}^{j}, p\right)$ (ordered by non-decreasing request duration, akin to $w c l x^{>}\left(T_{x}, T_{i}^{j}\right)$ ), and let $s=\left|w \operatorname{csp}\left(T_{i}^{j}, p\right)\right|, b=n d b p\left(T_{i}^{j}, p\right)$, and $\left(\mathcal{R}_{l}, k_{l}\right)=$ $w \operatorname{csp} p_{l}^{>}\left(T_{x}, T_{i}^{j}\right)$, then a bound for the interference from short requests executed on $p$ not accounted for in $\operatorname{rbl} l p\left(T_{i}^{j}, p\right)$ is given by

$$
\operatorname{rbsp}\left(T_{i}^{j}, p\right)=\sum_{l=1}^{\min (b, s)}\left|\mathcal{R}_{l}\right| .
$$

\section{A.7 A Tighter Bound for $\mathrm{SB}\left(T_{i}^{j}\right)$}

The intuitive and simple bound for $\mathrm{SB}\left(T_{i}^{j}\right)$ stated above is likely to be sufficient in most scenarios where jobs make few short requests. However, a tighter bound can be obtained by following an approach similar to that used for bounding $\mathrm{LB}\left(T_{i}^{j}\right)$. We define $\operatorname{wcsx} g\left(T_{x}, T_{i}^{j}, g\right)$ similarly to $\operatorname{wcs} x\left(T_{x}, T_{i}^{j}\right)$ to denote short requests made by jobs of $T_{x}$ while $T_{i}^{j}$ is pending that involve resources in group $g$ (note that, compared to $\operatorname{wcs} x\left(T_{x}, T_{i}^{j}\right)$, the $\operatorname{subreq}(\mathcal{R})$ constraint is absent):

$$
\operatorname{wcsxg}\left(T_{x}, T_{i}^{j}, g\right)=\left\{(\mathcal{R}, k) \mid \mathcal{R} \in \operatorname{WCSR}\left(T_{x}\right) \wedge g=\mathrm{G}(\operatorname{res}(\mathcal{R})) \wedge k \in\left\{1, \ldots,\left\lceil\frac{\mathrm{p}\left(T_{i}\right)}{\mathrm{p}\left(T_{x}\right)}\right\rceil+1\right\}\right\} .
$$

Based on $\operatorname{wcsxg}\left(T_{x}, T_{i}^{j}, g\right)$, we define similarly to $\operatorname{wcsp}\left(T_{i}^{j}, p\right)$

$$
\operatorname{wcspg}\left(T_{i}^{j}, p, g\right)=\bigcup_{T_{x} \in \operatorname{partition}(p)} \operatorname{wcsxg}\left(T_{x}, T_{i}^{j}, g\right) .
$$

Let $s=\left|\operatorname{wcspg}\left(T_{i}^{j}, p, g\right)\right|, b=\left|\left\{\mathcal{R} \mid \mathcal{R} \in \mathrm{SR}\left(T_{i}^{j}\right) \wedge g=\mathrm{G}(\operatorname{res}(\mathcal{R}))\right\}\right|$, and $\left(\mathcal{R}_{l}, k_{l}\right)=\operatorname{wcspg}_{l}^{>}\left(T_{i}^{j}, p, g\right)$ (akin to $\left.\operatorname{wcsp}_{l}^{>}\left(T_{x}, T_{i}^{j}\right)\right)$. A bound for direct blocking through group $g$ on processor $p$ is given by

$$
\operatorname{sbgp}\left(T_{i}^{j}, g, p\right)=\sum_{l=1}^{\min (b, s)}\left|\mathcal{R}_{l}\right|
$$

and hence a bound across all processors is given by

$$
\operatorname{sbg}\left(T_{i}^{j}, g\right)=\sum_{\substack{p=1 \\ p \neq \mathrm{P}(t s k(\mathcal{R}))}}^{m} \operatorname{sbgp}\left(T_{i}^{j}, g, p\right) .
$$

A bound on $\mathrm{SB}\left(T_{i}^{j}\right)$ follows:

$$
\mathrm{SB}\left(T_{i}^{j}\right)=\sum_{g \in\left\{\mathrm{G}(\mathcal{R}) \mid \mathcal{R} \in \mathrm{SR}\left(T_{i}^{j}\right)\right\}} \operatorname{sbg}\left(T_{i}^{j}, g\right)
$$

This bound yields improved blocking terms if $T_{i}^{j}$ issues frequent requests to few groups. When comparing the FMLP to other synchronization protocols, the improved bound given in Equation 28 should be used.

\section{A.8 Obtaining Per-Task Bounds}

Up to this point, blocking bounds have been derived for a specific job $T_{i}^{j}$. The advantage of this approach is that jobspecific behavior ( $\operatorname{LR}\left(T_{i}^{j}\right)$ and $\operatorname{SR}\left(T_{i}^{j}\right)$, if they are known) can be used to obtain a better per-job bound. However, per-task bounds are often required for schedulability analysis. Per-task bounds, i.e., bounds that limit worst-case blocking for any job of $T_{i}$, can be obtained as follows. If a task is known to release only a finite number of jobs, then one can simply find the maximum of the per-job bounds. If a task releases a potentially infinite sequence of jobs, then one can derive per-task bounds by replacing both every occurrence of $\operatorname{LR}\left(T_{i}^{j}\right)$ with $\operatorname{WCLR}\left(T_{i}\right)$ in Equations 7, 16, and 17 as well as every occurrence of $\operatorname{SR}\left(T_{i}^{j}\right)$ with $\operatorname{WCSR}\left(T_{i}\right)$ in Equations 13, 26, and 28. 


\section{A.9 Bounding Deferral Blocking: $\mathrm{DB}\left(T_{i}\right)$}

The standard technique for bounding deferral blocking is to charge the minimum of the execution cost and self-suspension time for every local higher-priority task [20]. Under the FMLP, a bound on the maximum time that a job $T_{r}^{k}$ self-suspends is given by $\operatorname{LB}\left(T_{r}^{k}\right)$. Hence, the maximum self-suspension time of $T_{r}$ is given by

$$
\operatorname{lbt}\left(T_{r}\right)=\max \left(\left\{\operatorname{LB}\left(T_{r}^{k}\right) \mid T_{r}^{k} \in j o b s\left(T_{r}\right)\right\}\right) .
$$

Note that $l b t\left(T_{r}\right)$ can be computed as described in Sec. A.8. The standard bound for deferral blocking hence is given by

$$
\mathrm{DB}\left(T_{i}\right)=\sum_{\substack{x<i \\ \mathrm{P}\left(T_{x}\right)=\mathrm{P}\left(T_{i}\right)}} \min \left(\mathrm{e}\left(T_{x}\right), \operatorname{lbt}\left(T_{x}\right)\right)
$$

\section{A.10 Inflating Request and Execution Times to Account for Spinning}

Prior to both computing $\mathrm{DB}\left(T_{i}\right)$ and testing schedulability, $\mathrm{e}\left(T_{x}\right)$ must have been inflated to account for execution time lost to spinning. Similarly, the request duration of long requests that contain nested short requests must be inflated to account for the nested blocking. Correct results can be obtained by computing blocking terms as follows:

1. Compute blocking times for all short requests with Equation 12.

2. Inflate all worst-case request durations of long requests that contain short requests to account for spinning as determined by Equation 12. Note that the improved bound given in Equation 28 cannot be used in this step since it does not yield per-request blocking bounds, which are required to safely inflate outer long requests.

3. Inflate all worst-case execution costs to account for spinning as determined by Equation 28 . The improved bound can be used in this step since only the overall impact from all short requests made by a job is required.

4. Compute $\mathrm{B}\left(T_{i}\right)$ for each task $T_{i}$. Recall that per-task bounds can be computed as described in Sec. A.8. 